\title{
Silk Fibroin-Carbon Nanotube Composites based Fiber Substrated Wearable Triboelectric Nanogenerator
}

\author{
Meng Su, ${ }^{\dagger}{ }^{*}$ Beomjoon $\mathrm{Kim}^{\dagger}$ \\ $\dagger$ CIRMM, Institute of Industrial Science, The University of Tokyo, Tokyo 153-8505, Japan. \\ * Corresponding author. E-mail addresses: sumeng@iis.u-tokyo.ac.jp
}

Material selection of silk fibroin and carbon nanotube. Silk is natural degradable skin-friendly material, very light in weight, comfortable to wear, and breathable. Because silk fibroin is a natural animal protein, it is very easy to process and mix with other substances. Other materials that have better power generation performance ("+" positive side) are inferior in terms of softness and workability; Multi-walled carbon nanotube is one of the most inexpensive choice for non-metallic conductive material. The component is simple and pure, and the commercialized powder provides much convenience in the following metering and mixing experiments.

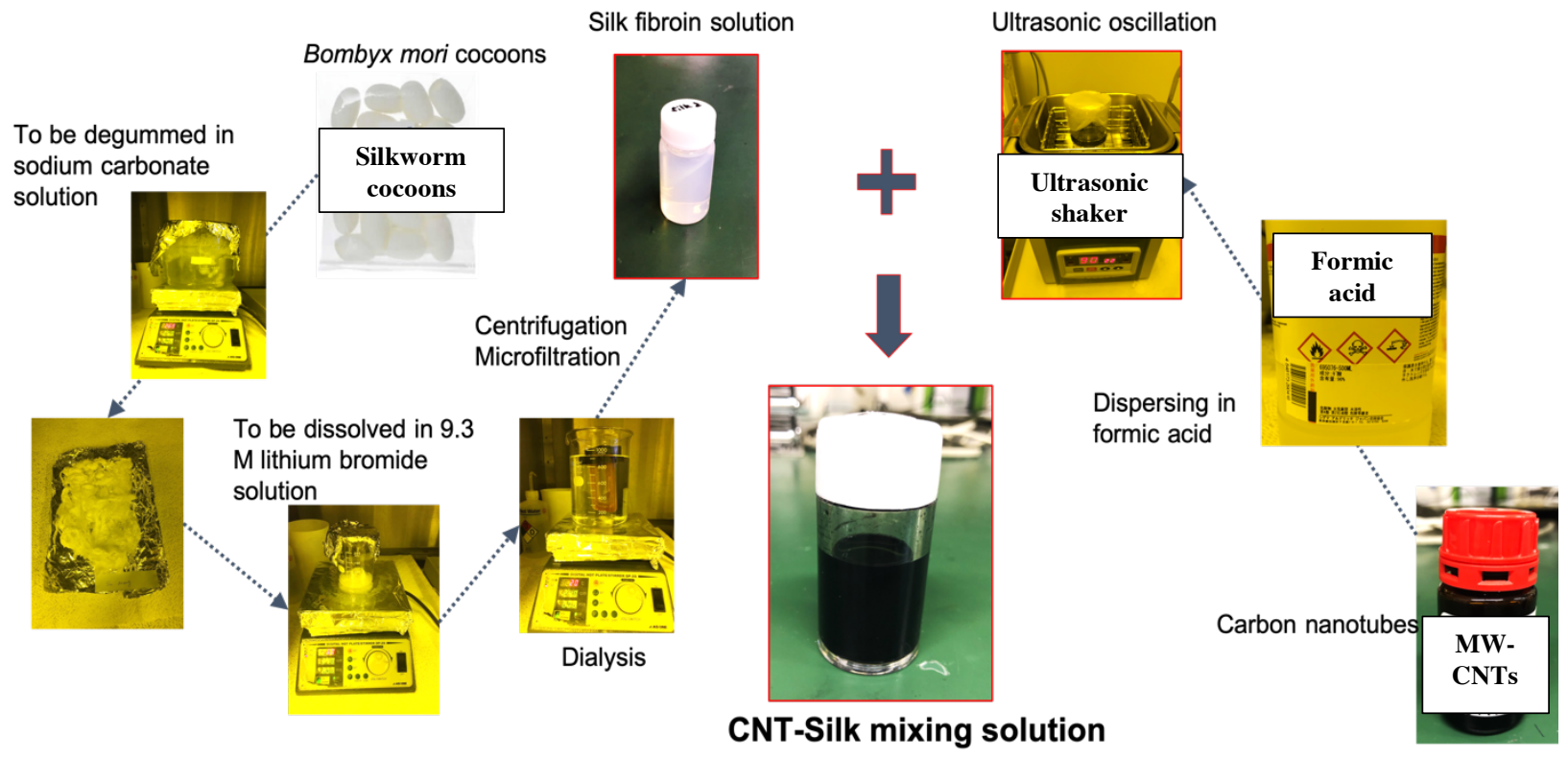

Figure S1. Bombyx mori cocoons were boiled in $0.02 \mathrm{M}$ sodium carbonate solution for $45 \mathrm{~min}$ to be degummed. The silk fibers obtained were then rinsed and allowed to air dry at room temperature for $24 \mathrm{~h}$. Next, the silk fibers were dissolved in 9.3 M lithium bromide ( $\mathrm{LiBr}$ ) solution. Then, the LiBr was removed by dialysis (3.5 K MWCO, Slide-A-Lyzer Dialysis Cassette, Thermo Fisher Scientific Inc.) for $48 \mathrm{~h}$. Next, the silk fibroin solution was purified by centrifugation followed by microfiltration. In our case, $4.2 \mathrm{wt} \%(40 \mathrm{mg} / \mathrm{ml})$ silk fibroin solution was obtained. 
Finally, the silk fibroin solution was added to the CNT-formic acid solution, then shaken for 2 hours under ultrasound bath to obtain the silk fibroin-CNT-formic acid mixing solution.

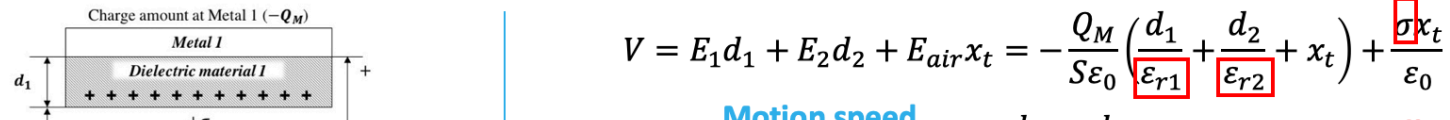

$$
\begin{aligned}
& \text { Motion speed } \quad \frac{d_{1}}{\varepsilon_{r 1}}+\frac{d_{2}}{\varepsilon_{r}} \quad \text { Material's intrinsic }
\end{aligned}
$$

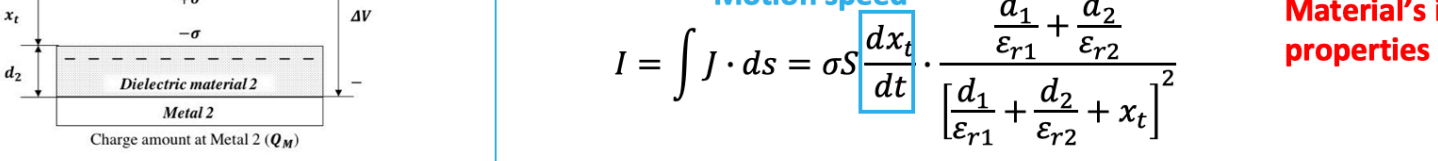

$$
\begin{aligned}
& V=E_{2} d_{2}+E_{\text {air }} x_{t}=-\frac{Q_{M}}{S \varepsilon_{0}}\left(\frac{d_{2}}{\frac{\varepsilon_{r 2}}{d_{2}}}+x_{t}\right)+\frac{\sigma x_{t}}{\varepsilon_{0}} \\
& I=\int J \cdot d s=\sigma S \frac{d x_{t}}{d t} \cdot \frac{\frac{d_{1}}{\varepsilon_{r 1}}+\frac{d_{2}}{\varepsilon_{r 2}}}{\left[\frac{d_{1}}{\varepsilon_{r 1}}+\frac{d_{2}}{\varepsilon_{r 2}}+x_{t}\right]^{2}} \\
& V=-\frac{Q_{M}}{C}+V_{O C} \\
& =-\frac{1}{w \varepsilon_{0}\left(l-x_{t}\right)}\left(\frac{d_{1}}{\frac{\varepsilon_{r 1}}{\varepsilon_{r 2}}}+\frac{d_{2}}{\varepsilon_{r 2}}\right) Q_{M}+\frac{\sigma x_{t}}{\varepsilon_{0}\left(l-x_{t}\right)}\left(\frac{d_{1}}{\varepsilon_{r 1}}+\frac{d_{2}}{\varepsilon_{r 2}}\right) \\
& I=\frac{d Q_{M}}{d t}=\sigma w \cdot \frac{d x}{d t}
\end{aligned}
$$

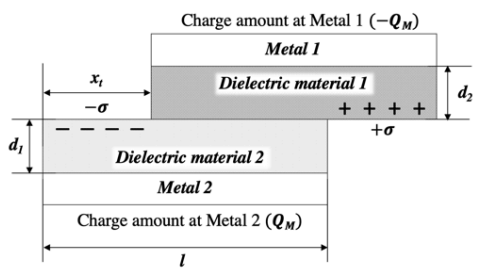

$S$-- the contact area

$\varepsilon_{0}$-- the vacuum permittivity of air

$\varepsilon_{r}$-- the vacuum permittivity of dielectric material $\sigma--$ charge density

$J$-- current density

$C$-- capacitance of TENG

$w$-- width of dielectrics

Figure S2. It can be known that the magnitude of the output voltage and current both have a great relationship with the charge density $\sigma$, materials permittivity $\varepsilon_{r}$, which are the intrinsic material properties, and surface morphology. In addition, the relative movement speed $d x_{t} / d t$ and distance $d$ between two dielectrics also affects the output.

Generally, in actual wear, the relative distance between friction objects is orders of magnitude far greater than the thickness of the friction material itself, and the too large relative movement distance will make this effect negligible, so this factor $d$ is far less important than the ability of the friction material to generate charges. As for the contact speed $d x_{t} / d t$, indeed, the higher the average speed, the higher the output. Considering that in real life, the friction of the human body is mostly slight and continuous within a certain period of time, such as walking, writing, tapping a keyboard, et al, so the frequency of friction can indirectly reflect the friction speed. However, in order to fit reality, in the following experiments, low-frequency vibration is still used for power generation detection.

Based on the discussions, the main points about the experimental design are summarized as follows:

(1) The amplitude of the external vibration should be several orders of magnitude higher than the thickness of the friction material $\left(x_{t}>500 \mu m, d \approx 40 \sim 100 \mu\right)$

(2) Using low frequency vibration of $<10 \mathrm{~Hz}$ for power generation test $(f=7 \mathrm{~Hz}$ ) 
(3) The friction material of the TENG is selected to have a strong power generation capability and has excellent dielectric properties $\left(\varepsilon_{r(s i l k)}=6.1>\varepsilon_{0}=1\right)$

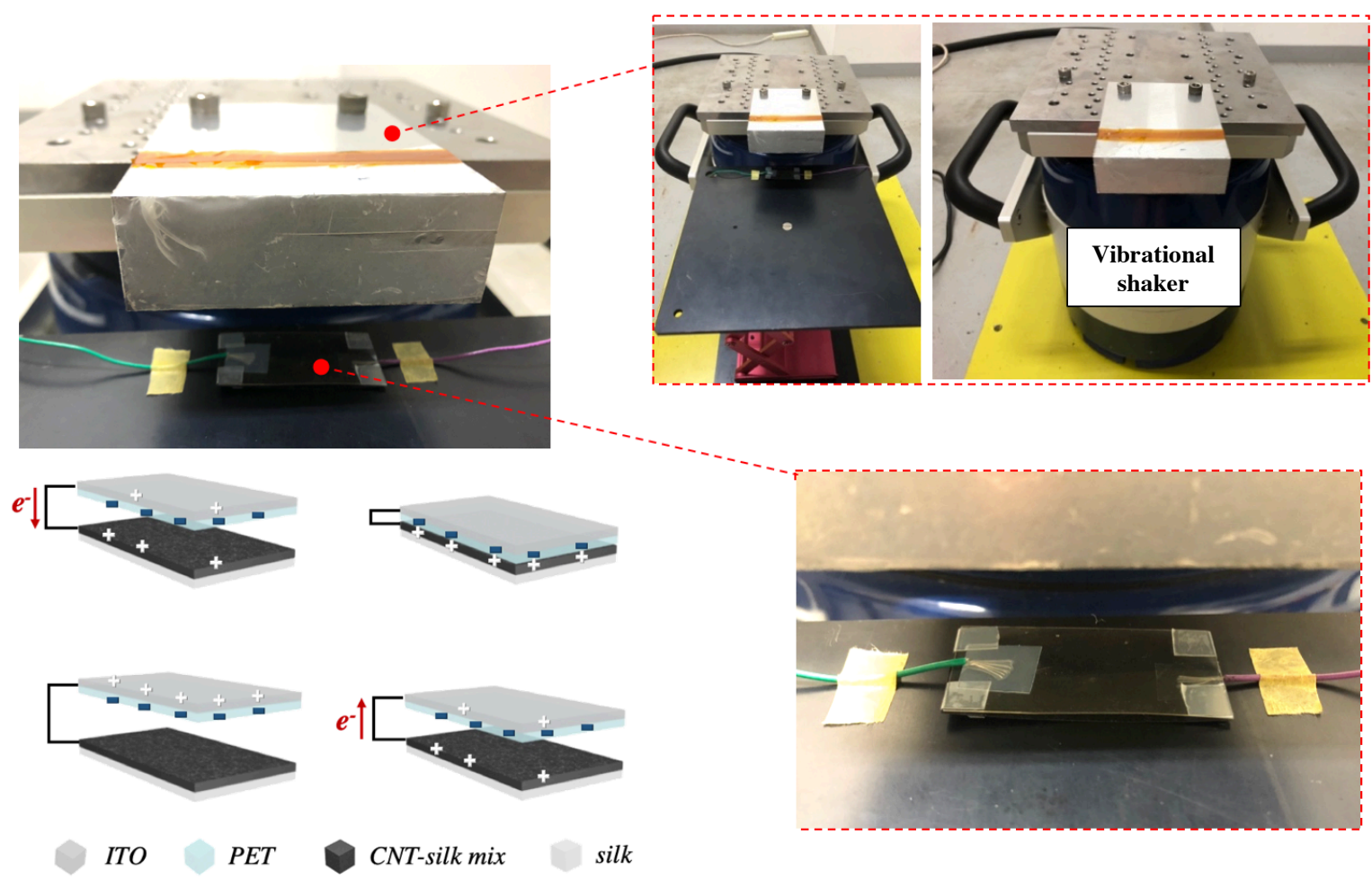

Figure S3. A vibration platform was set up for studying on electrical properties of the fabricated silk-CNT TENG. Firstly, a functional generator (SG-4105, IWATSU Electric Co., Ltd) was used to generate a $7 \mathrm{~Hz}$ electrical signal with an amplitude of $1.3 \mathrm{~V}$ which was subsequently enlarged by an amplifier (MA1, IMV Corp.). Secondly, the amplified signal was utilized to drive a shaker (m060, IMV Corp.), and then a periodic vibration with the frequency of $7 \mathrm{~Hz}$ and amplitude of 35 $\mathrm{mm}$ was obtained. An aluminum mass was fixed on the shaker to provide an external force to the silk-CNT TENG placed underneath the mass. 


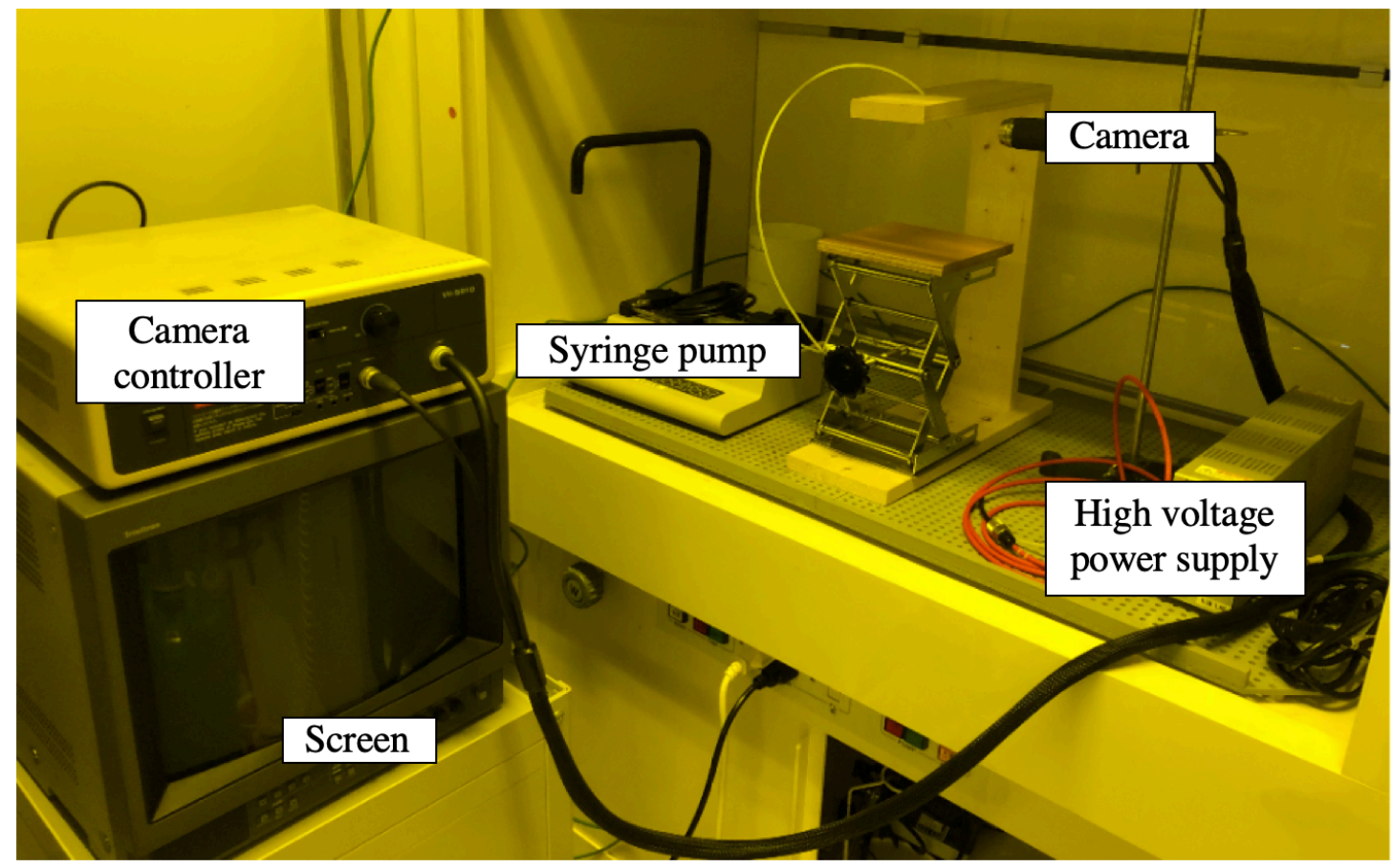

Figure S4. The setup used in electrospinning and electrospray experiments. The high voltage power supply (Model HJPQ-30PI, Matsusada) was connected with the needle tip, from where the droplet will be pumped out. Using the camera connected to the screen, the form of the droplet could be observed in real time. The applied voltage and pumping rate would be adjusted based on the condition of the droplet, to keep the Taylor cone stable. 

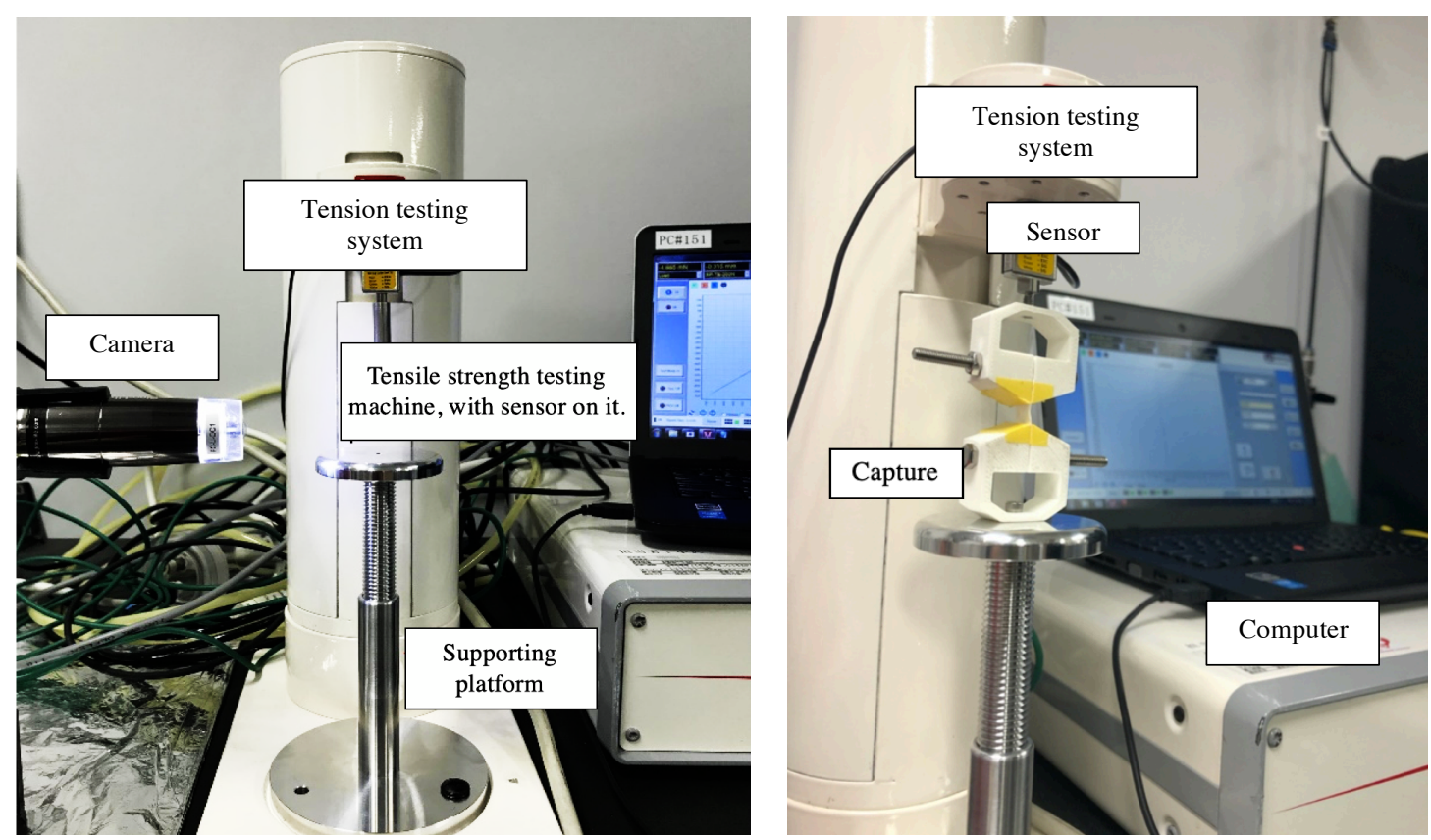

Figure S5. The setup used in tensile strength testing experiments. The dynamic tension testing system (Reimagine TE200 Testing systems, Illinois Tool Works) itself has a matched sensor. The supporting platform and captures were designed and manufactured according to the properties of the machine and samples. The computer could control the rising speed of the upper fixture. When the sample reached the tensile limit, the sensor was pulled and a curve of tensile force was formed on the computer. 
(a)

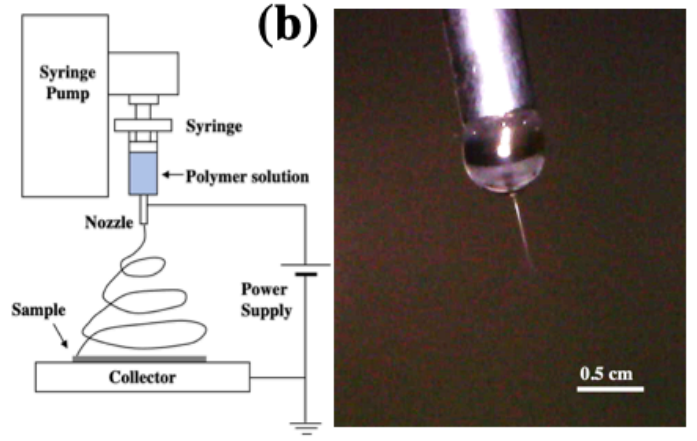

(d)

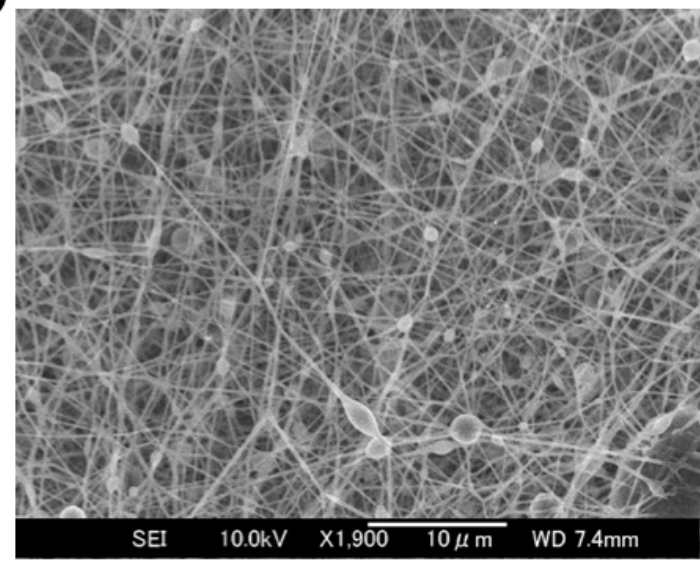

(c)

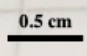

Thickness: $107 \mu \mathrm{m}$

(e)

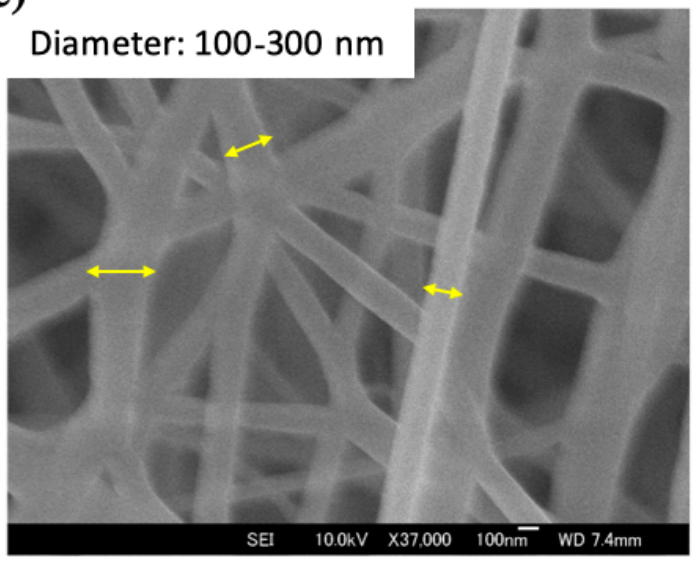

Figure S6. The electrospinning solution was prepared by mixing silk fibroin solution and poly (ethylene oxide) (PEO) in the weight ratio between silk fibroin and PEO of 2:1 in a formic acid solvent, to achieve $13 \mathrm{wt} \%$. The electrospinning process was conducted by using a setup shown in (a). During experiments, the temperature was $22-27^{\circ} \mathrm{C}$, and the relative humidity was $40-60 \%$. Electrospinning for 30 minutes produced an about 200-micron-thick film of $2 \times 4 \mathrm{~cm}^{2}$. The experiments were mostly completed during the rainy season, so the experimental environment was very humid. Although humidity was said to have an effect on the stability of the electric field, according to these successful experiences, the importance of other parameters, such as flow rate, voltage, and solution viscosity, should be much higher than humidity. The diameter of the spun fibers was between 100-300 nanometers as shown in (d-e), and some nodes could be seen to be generated, which were speculated to be due to the effect of too high humidity. 
(a)

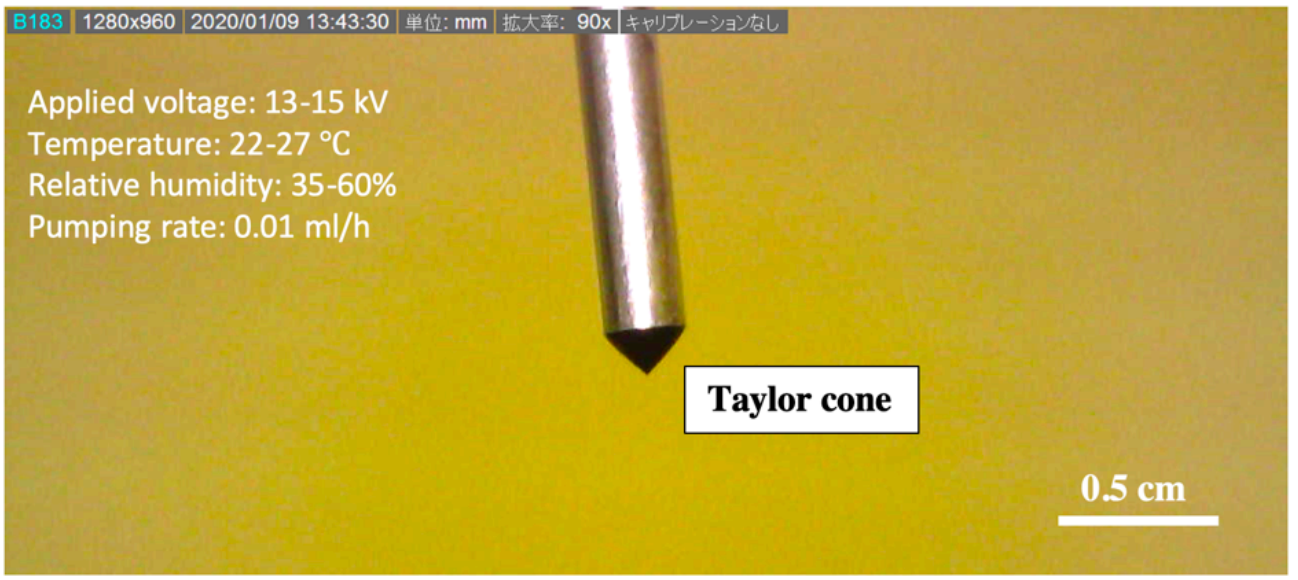

(b)
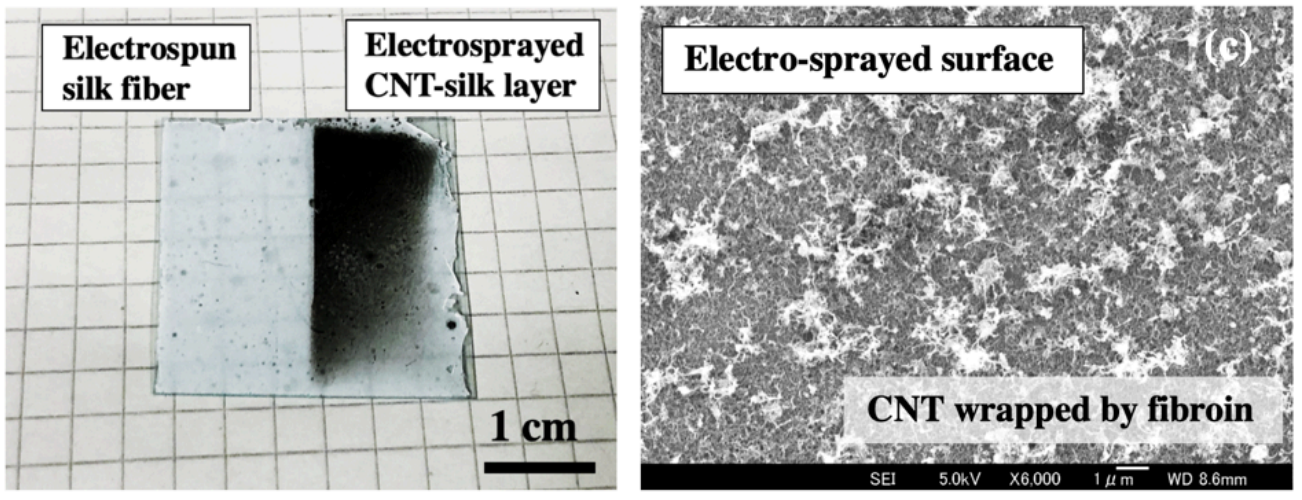

(d)
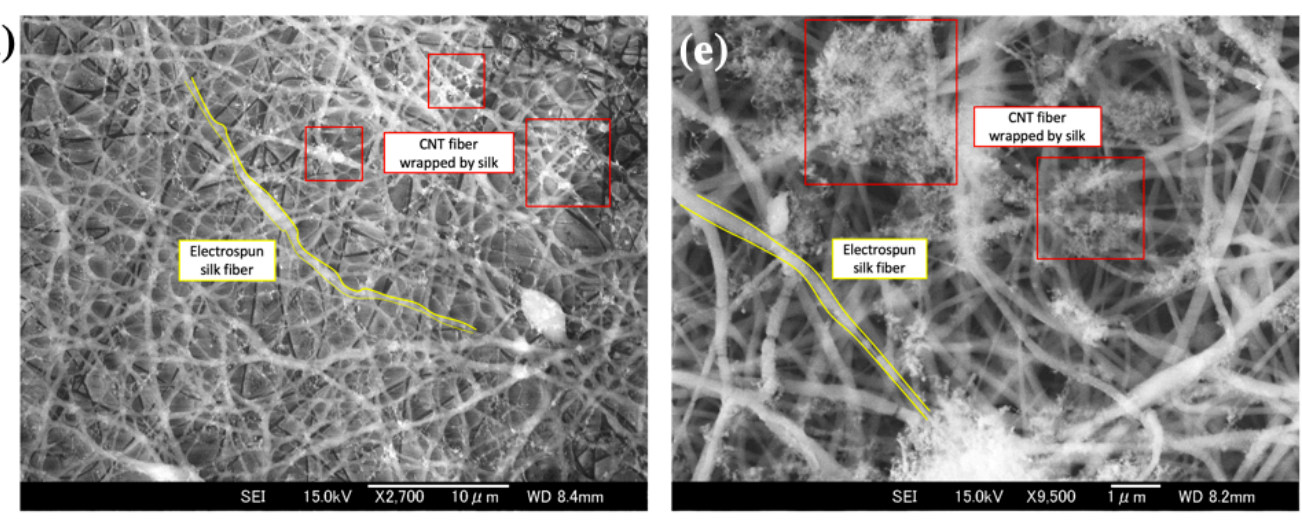

Figure S7. The electrospray solution was the CNT-silk solution without any extra processing. The setup was the same as that used in electrospinning. Because formic acid as a solvent corrodes the fiber structure of the silk substrate, the size of the sprayed droplets must be strictly controlled at the nanometer level. Only in this way can the solvent be rapidly evaporated during the dropping process and not be carried to contaminate the substrate. Therefore, the applied voltage was set as 13-15 kV, controlled manually to form a stable Taylor cone. Because the droplets produced by electrospray were too small and the pumping rate was too slow, it was difficult to observe them with a magnifying lens like electrospinning, as shown in (a). Therefore, we could only rely on whether the Taylor cone was formed steadily to judge whether electrospray was successfully 
performed. The morphology of the droplets can hardly be observed on the accumulated sprayed layer surface, and only the appearance of silk wrapped with CNTs could be seen, as shown in (c). When the sprayed amount was relatively small, the joint part of the two layers could still be observed. The silk fiber has a diameter of nanometers, which allows the carbon nanotubes to be entangled with silk fibers or filled in the gaps between the fibers that are also nanoscale, as shown in (d-e). Although it has not been compared with substrates of other materials, the adhesion of the sprayed layer on the parts other than the substrate was poor during experiments. So the electrospun substrate is speculated to be necessary for holding the top layer.
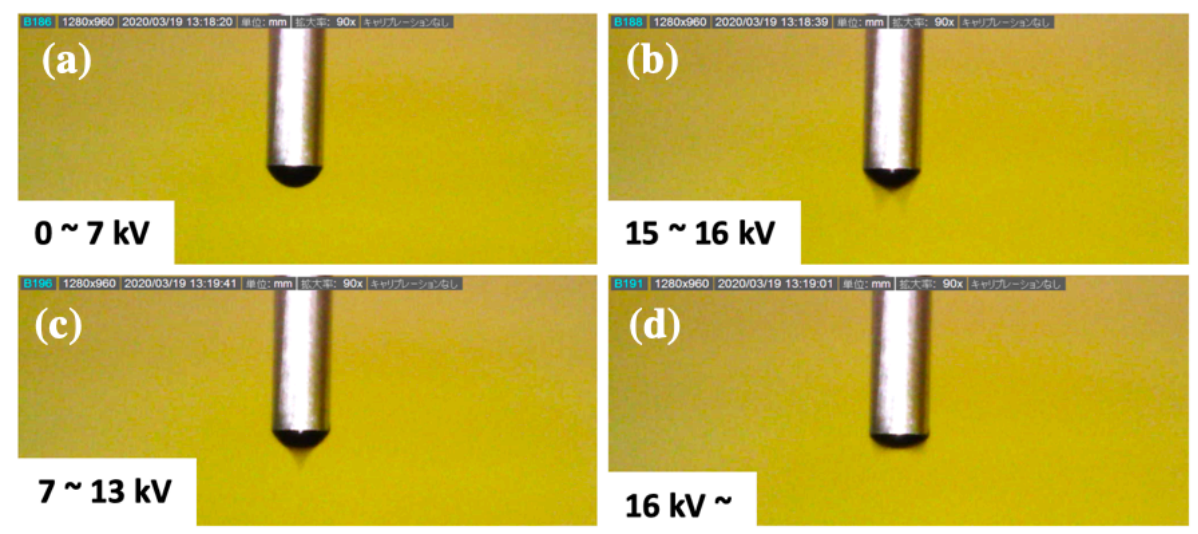

Figure S8. The mixing solution has not been applied to electrospray before, so knowing the information about bad conditions is also important. (a) When the electric field was not strong enough, the droplet was naturally round or had a stable translucent cone. (c) As the voltage continued to increase, a completely opaque cone would appear. Only then would electrospray be carried out. (b,d) If the voltage was too high, the translucent cone would reappear and highly unstable. the consequences of the bad conditions were all just the same, that is, large-diameter droplets destroy the fiber substrate. 
(a) Shaker Patting

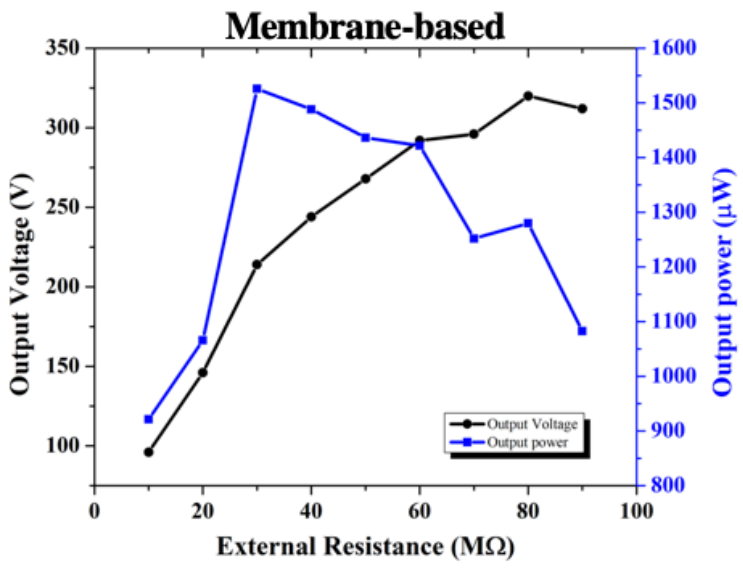

Fiber-substrated

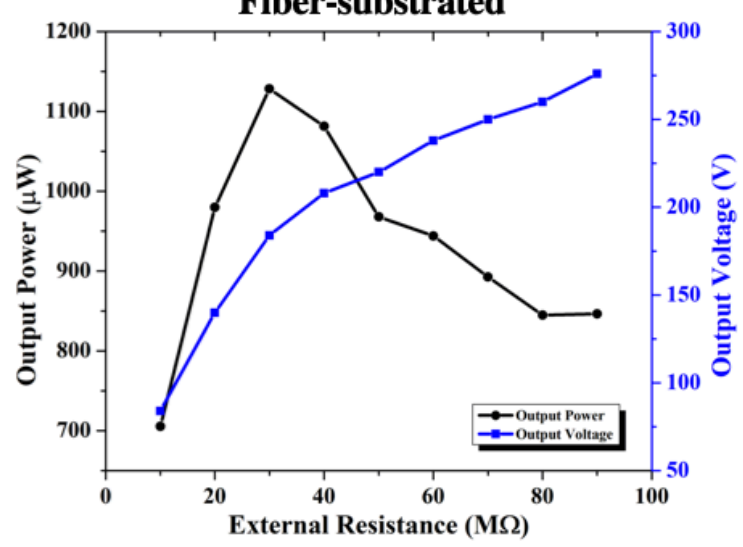

(b) $\underline{\text { Hand Patting }}$

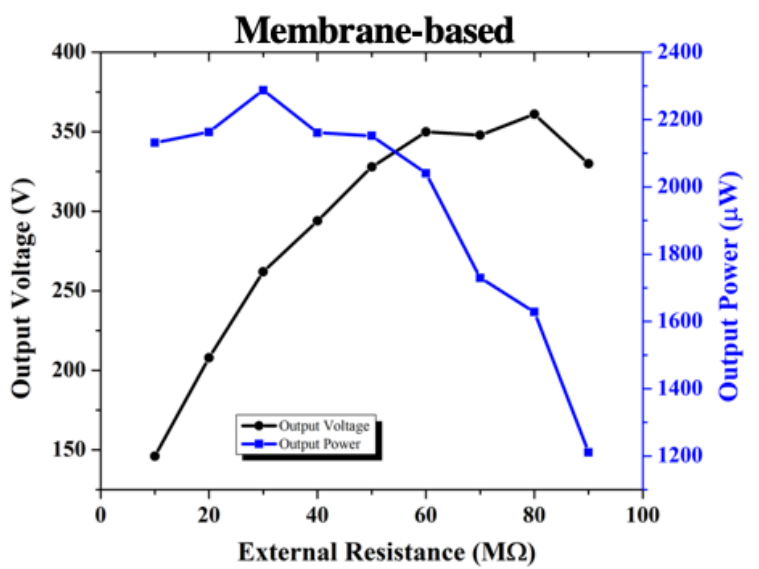

Fiber-substrated

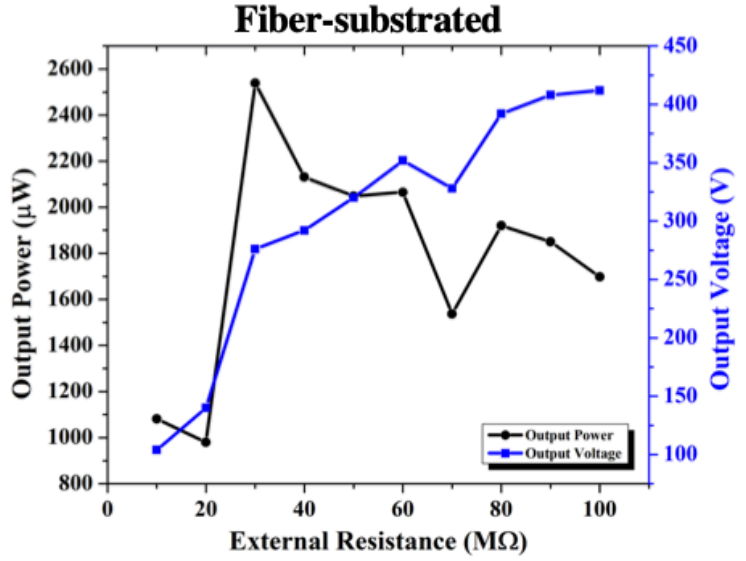

Figure S9. Under shaker patting, the previous membrane-based TENG's peak output power reached $190.73 \mu \mathrm{W} / \mathrm{cm}^{2}$, the fiber-substrated TENG reached $140.99 \mu \mathrm{W} / \mathrm{cm}^{2}$. Under hand patting, the membrane-based TENG's output power reached $285.91 \mu \mathrm{W} / \mathrm{cm}^{2}$, and fiber-substrated TENG reached $317.4 \mu \mathrm{W} / \mathrm{cm}^{2}$. Compared with the previous CNT-silk membrane TENG with the same structure and the same experimental conditions, under shaker patting, the peak output power was not very different from the membrane based case; under hand patting, this output was higher than the membrane based TENG, speculated to be due to more complex microstructures. 


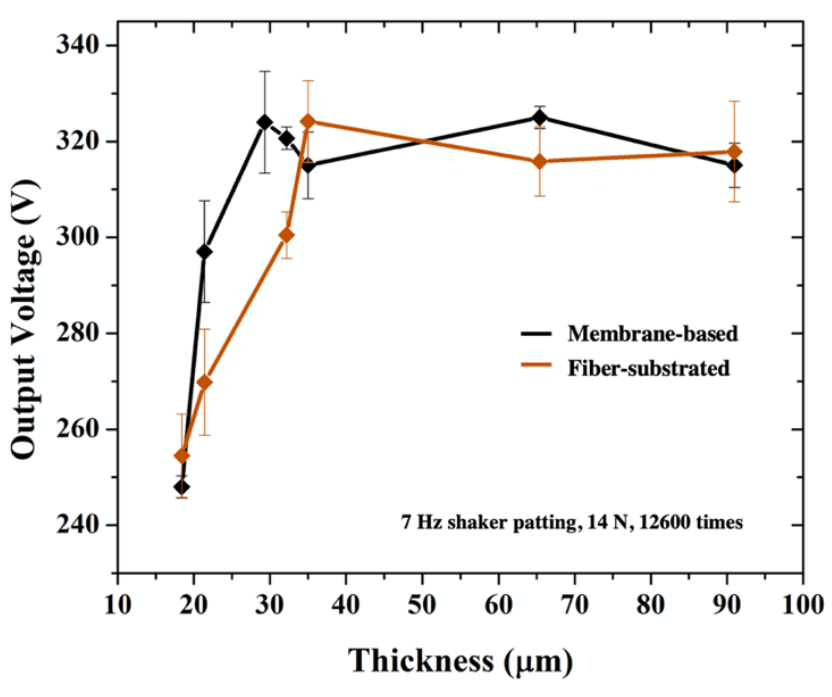

Figure S10. The effect of thickness on output characteristics have been explored. It can be seen from the results that after the thickness of the membrane-based TENG reaches $30 \mu \mathrm{m}$, the increase in thickness will not significantly affect the output. Although there are some fluctuations in the output curve in the figure, they are basically within $5 \%$, which can be ignored. In the case of fiber-substrated TENGs, the thickness of the mixing layer stabilizes after reaching $40 \mu \mathrm{m}$. In the stated experiments, the thickness of the membrane-based TENG in the case of

the PET testing TENG was about $50 \mu \mathrm{m}$, and the case of independent films was about $100 \mu \mathrm{m}$; for the fiber-substrated TENG, both of the thickness of the PET testing samples and independent fiber samples are around 40 50 $\mu \mathrm{m}$. Because they are all in the stable and safe range, the obtained results have been proven to be effective and reliable.

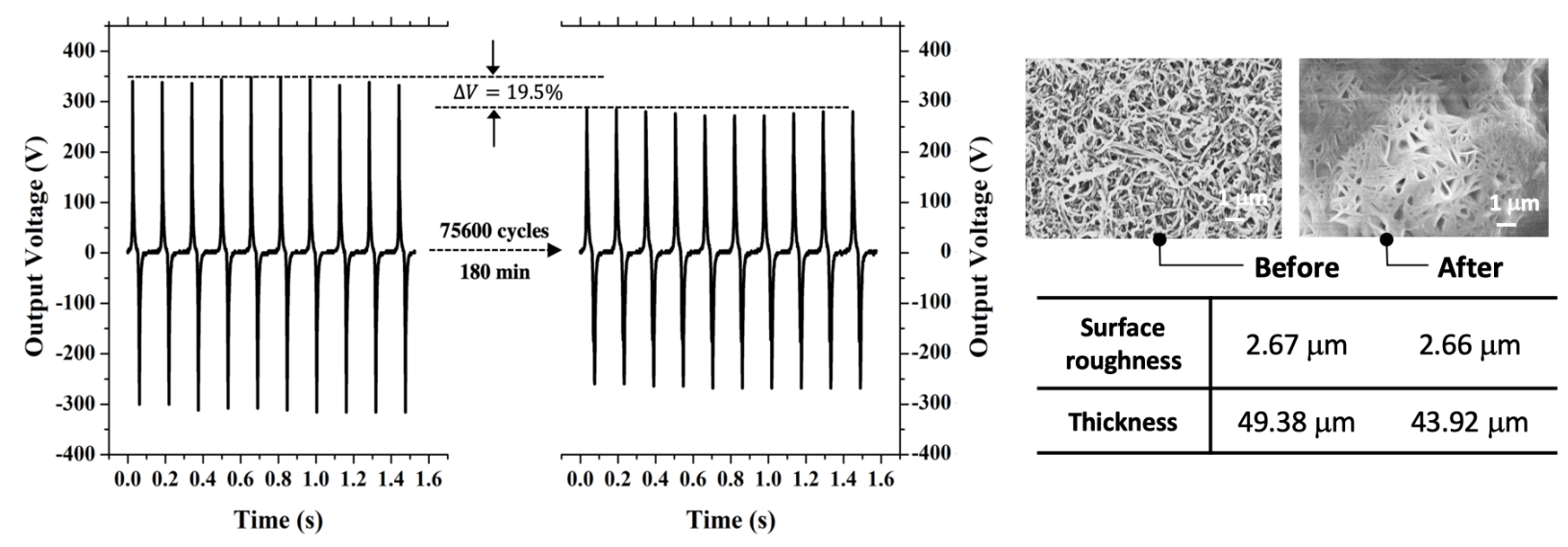

Figure S11. The durability of the F-TENG was also tested for continuous operation by using the vibration platform for $180 \mathrm{~min}$. After 75600-cycle of beating with an external force, the output voltage of the TENG was almost unchanged with a slight decrease of $16.7 \%$. In daily lives, the average number of walking steps per person per day is about 8000 steps, and about 50,000 to 60,000 steps in a week. So, in this study, the initially established durability goal is that there are no destructive defects for more than 50,000 cycles of work. In different parts of the human body, the mode of friction is different, so there are certain differences in the standards for the working cycles that can be tolerated. In this experiment, the number of valid working cycles verified was more than 50\% higher than the target and was evaluated as being able to cover the deviations caused by different friction modes on different parts of the human body. Therefore, as a conclusion, the testing results show that the durability of the TENG has satisfied the preset target. 